\title{
A Case of Chronic Wasting Disease in an Elk Imported to Korea from Canada
}

\author{
Hyun-Joo SOHN ${ }^{1}$, Jae-Hoon $\mathrm{KIM}^{1) *}$, Kang-Seuk CHOI ${ }^{1}$, Jin-Ju NAH ${ }^{1}$, Yi-Seok JOO ${ }^{1)}$, Young-Hwa JEAN ${ }^{1)}$, \\ Soo-Whan $\mathrm{AHN}^{1)}$, Ok-Kyung $\mathrm{KIM}^{1)}$, Dae-Yong $\mathrm{KIM}^{2)}$ and Aru BALACHANDRAN ${ }^{3)}$ \\ ${ }^{1)}$ National Veterinary Research and Quarantine Service, Anyang 430-824, ${ }^{2)}$ Department of Pathology, College of Veterinary Medicine, \\ Seoul National University, Suwon 441-744, Korea and ${ }^{3)}$ Animal Disease Research Institute, Canadian Food Inspection Agency, Nepean, \\ Ontario, Canada
}

(Received 13 March 2002/Accepted 8 May 2002)

\begin{abstract}
A seven-year-old male elk (Cervus elaphus nelsoni) was euthanized and necropsied after having a 3-week history of body weight loss, emaciation, excessive salivation, teeth grinding, fever, anorexia, and respiratory distress. The elk was imported into Korea from Canada on March 9, 1997. Gross pathologic findings were restricted to a diffuse fibrinous pneumonia. Microscopic lesions included mild neuronal vacuolation and spongiform change in the neuropil of selected brain stem nuclei and generalized astrocytosis. Immunohistochemistry for protease-resistant prion protein $\left(\mathrm{PrP}^{\mathrm{res}}\right)$ was positive in all brain sections but more pronounced in the section of the obex of the medulla. And the $\operatorname{PrP}^{\text {res }}$ was also detected by western immunoblotting in the brain and spinal cord. All the remaining elk and deer that had been in contact with this elk were destroyed and negative for chronic wasting disease (CWD). To our knowl edge, this is the first case of CWD occurring outside of the U.S.A. and Canada.

KEY WORDS: chronic wasting disease, elk, immunohistochemistry.
\end{abstract}

Chronic wasting disease (CWD), a fatal neurologic disease with abnormal behavior, was first recognized in 1967 in captive mule deer (Odocoileus hemionus) and mule deer $\mathrm{x}$ white tailed deer (Odocoileus hemionus virginianus) hybrids held at wildlife facilities in Colorado, U.S.A. [8]. Subsequently similar disease conditions were also found in black-tailed deer (Odocoileus hemionus columbianus) and Rocky Mountain elk (Cervus elaphus nelsoni) in southeastern Wyoming, northeastern Colorado, and western Nebraska [8, 9]. CWD belongs to a member of a group of diseases collectively known as the transmissible spongiform encephalopathies (TSE), which are characterized by neuroparenchymal vacuolation and accumulation of a proteaseresistant isoform of prion protein. The characteristic clinical signs of CWD are severe emaciation, weight loss, unusual behavior such as lack of coordination, separation from the other animals in the herd, depression, paralysis, difficulties in swallowing, excessive salivation, and pneumonia [10]. Clinical signs last from several weeks to 8 months before the animal dies, but sometimes animals may not show any sign except for acute pneumonia or respiratory distress. Characteristic histopathologic change of CWD, spongiform encephalopathy, is found in brain stem, particularily the dorsal motor nucleus of the vagus nerve [10, 11].

CWD was first documented in a farmed elk (Cervus elaphus) in the province of Saskatchewan, Canada in 1996 and the affected elk was imported from the U.S.A. in 1989. In Korea, TSE has not been found in any animal species until the case described in this report. This paper describes a case of CWD in an imported elk diagnosed on the basis of clinical signs, histopathology, immunohistochemistry, and

\footnotetext{
* Correspondence to: Kim, J.-H., Pathology Division, National Veterinary Research \& Quarantine Service, Anyang 430-824, Korea.
}

immunoblotting. To our knowledge, this is the first case of CWD occurring outside of the U.S.A. and Canada.

A 7-year-old male elk died after a 3-week history of body weight loss, emaciation, excessive salivation, teeth grinding, fever, anorexia, and respiratory distress. The elk was imported from Canada on March 9, 1997 and had been kept in a farm that maintains a total of 40 elk and deer including 4 other elk that were imported from Canada in 1997. The elk had received no vaccination. Clinical signs were progressive despite antibiotics and fluid therapies.

Postmortem examination was performed by the referring veterinarian and the whole head and spinal cord were submitted to the National Veterinary Research \& Quarantine Service (NVRQS) for further examination. On necropsy, the elk was severely emaciated, and had a sunken eye and partial hair loss at right shoulder area. Tan to red sublobar or lobar consolidation was noted in the frontal and caudal lung lobes and there was fibrinous adhesion between the lung and the thoracic wall.

The brain and spinal cord were fixed in $10 \%$ neutral phosphate-buffered formalin, routinely processed, and stained with hematoxylin and eosin for light microscopic examination. Portions of the brain and spinal cord were frozen at $-70^{\circ} \mathrm{C}$ until analysis. Brain sections were taken from an obex, cerebellar peduncle, rostal colliculus, thalamus, midbrain, and frontal cerebral cortex. Paraffin blocks and slices of wet tissues were referred to the Animal Disease Research Institute, Nepean, Canada for confirmation.

For detection of protease-resistant prion protein $\left(\mathrm{PrP}^{\mathrm{res}}\right)$, immunohistochemical (IHC) staining method was performed as previously described [6]. Replicate paraffin sections of brain were pretreated with $88 \%$ formic acid for 5 min and rinsed in TB buffer (trizma base 29.8 g/D.W. 2L). The slides were then autoclaved at $121^{\circ} \mathrm{C}$ for $25 \mathrm{~min}$. After 

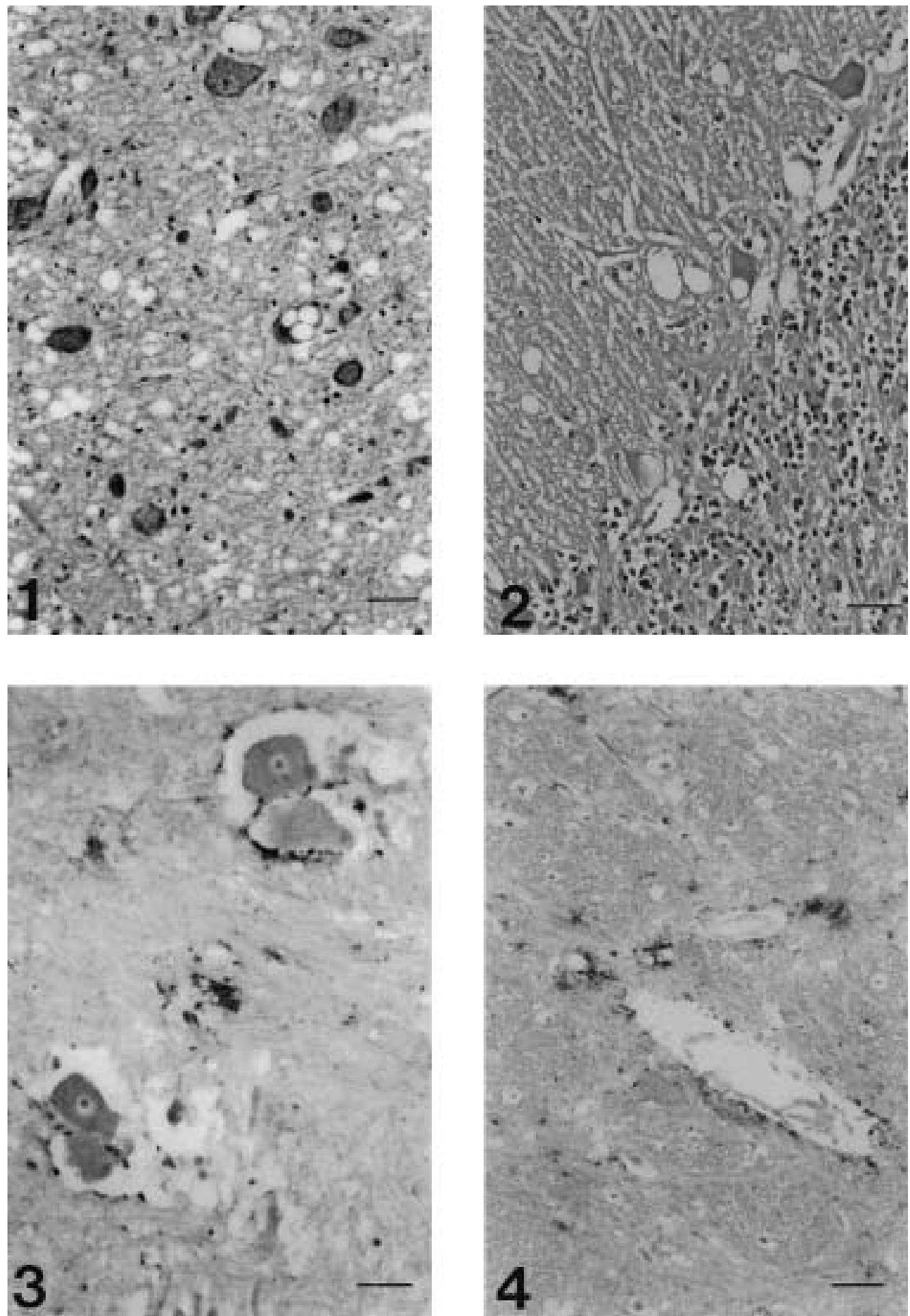

Fig. 1. Medulla oblongata. Note intracytoplasmic vacuoles in neuron and spongiform changes in the neuropils. HE. $\mathrm{Bar}=35 \mu \mathrm{m}$.

Fig. 2. Cerebellum. Note intracytoplasmic vacuoles in Purkinje's cell and spongiform changes in the Purkinje's cell layer. HE. Bar $=35 \mu \mathrm{m}$.

Fig. 3. Medulla oblongata. Note the extensive perinuronal and diffuse granular staining. PrP IHC. Bar=25 $\mu \mathrm{m}$

Fig. 4. Pons. Note the extensive perivascular and diffuse granular staining. PrP IHC. Bar=35 $\mu \mathrm{m}$. 


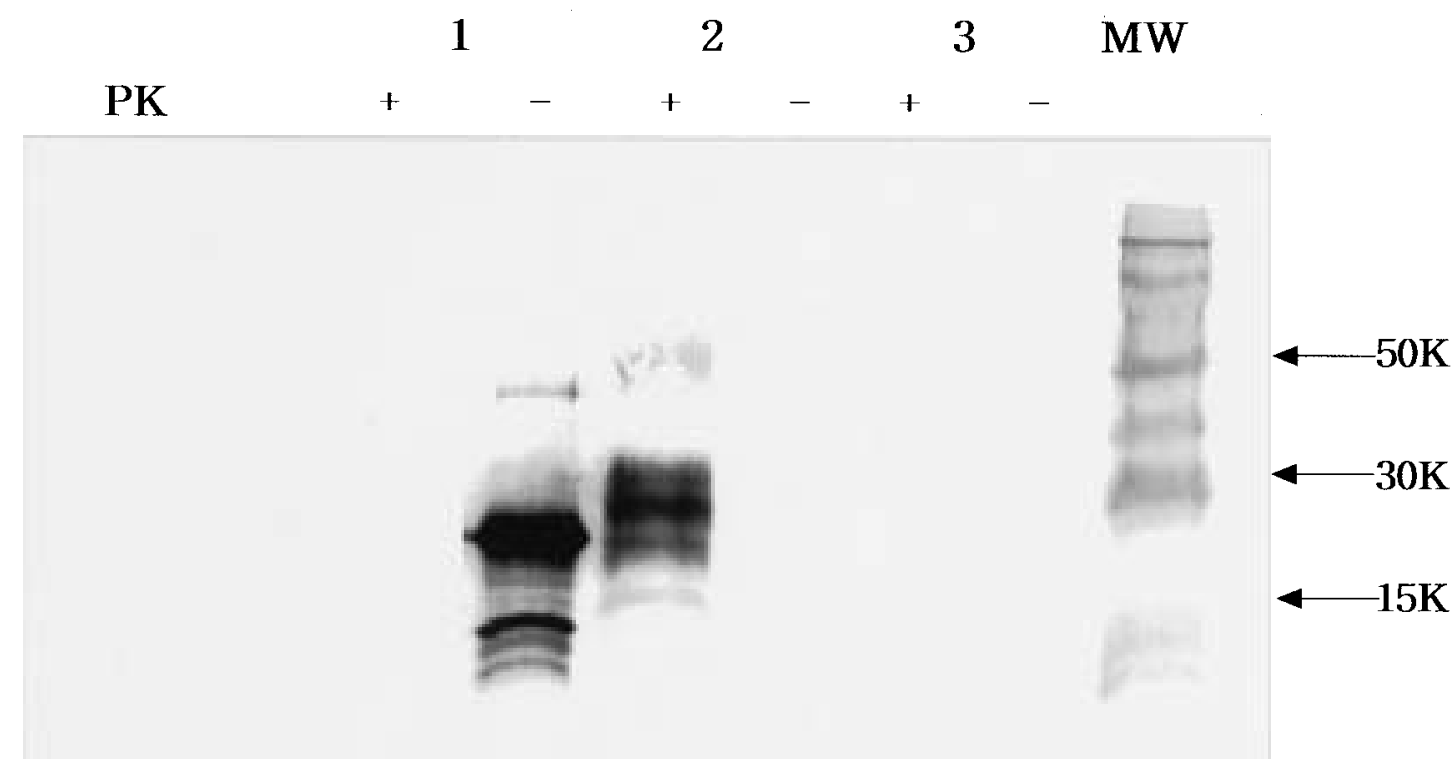

Fig. 5. Immunoblotting for the extracted samples of brain and spinal cord from elk: Samples were extracted with (+) or without (-) proteinase K treatment. Lane 1: positive control (recombinant PrP), Lane 2: Sample (Pool of spinal cord and brain from affected elk), Lane 3: Negative control (Normal elk brain).

incubating the slides with PrPres specific monoclonal antibodies (MAb) F89/160.1.5 and F99/97.6.1. (each diluted at $5 \mu \mathrm{g} / \mathrm{ml})[3,7]$ for overnight at $4^{\circ} \mathrm{C}$, the standard avidinbiotin-peroxidase complex (ABC) method (Vector Laboratories, Burlingame, CA, U.S.A.) was used according to the manufacturer's protocol to demonstrate the antigen using 3amino-9-ethylcarbazole (AEC) (Vector Laboratories, Burlingame, CA, U.S.A.) as the chromogen. CWD positive and negative control blocks were provided by Dr. Aru Balachandran at the Animal Disease Research Institute in Canada.

The western blotting method for detection of $\mathrm{PrP}^{\mathrm{res}}$ was performed using the pool of frozen spinal cord and brain. PrP was purified as previously described [5]. The purified PrP was subjected to electrophoresis on $12 \%$ polyacrylamide gels and then transferred to polyvinylidene difluoride (PVDF) membrane. After non-specific binding was blocked with $3 \%$ bovine serum albumin, the membrane was incubated with monoclonal antibodies F89/160.1.5 and F99/ 97.6.1. diluted at $5 \mu \mathrm{g} / \mathrm{ml}[3,7]$ and then with anti mouse IgG conjugated alkaline phosphatase (KPL, Maryland, U.S.A.). The membrane was finally stained with nitro blue tetrazolium chloride and 5-bromo-4-chloro-3-indolyl phosphate (NBT/BCIP) solution (KPL, Maryland, U.S.A.). Recombinant PrP expressed in E. coli BL21 was used as the positive control (Prionics AG, Switzerland).

Histopathologically, the brain and spinal cord were mildly autolyzed. Spongiform change, consisting of smaller vacuoles in the gray matter neuropil was observed in several brain stem nuclei. Single or small multiple vacuoles were observed in the peripheral cytoplasm of neurons (Fig. 1). The number of astrocytes was increased in most brain sections. In the cerebral cortex, angular, shrunken, hyperchro- matic neurons were often observed. But the occurrence of eosinophilic plaques was rare. Changes in cerebellar cortex consisted of focal spongiform change in molecular layer with prominent intracytoplasmic vacuolation of Purkinje cells (Fig. 2).

With immunohistochemistry, strong positive signals for $\mathrm{PrP}^{\mathrm{res}}$ were observed in the medulla oblongata, especially at the level of the obex. The positive reaction was pronounced in dorsal motor nuclei of the vagus nerve, spinal tract nucleus of the trigeminal nerve, olivary nucleus and the reticular formation. $\mathrm{PrP}^{\text {res }}$ positive signal was also detected in the red nucleus of midbrain, the pontine nucleus, and the greater thalamus nuclei. Outer granular layer of cerebral cortex and cerebellar cortex was more severely affected than the molecular layer.

The pattern of $\mathrm{PrP}^{\mathrm{res}}$ positive deposits observed included perineuronal aggregations (Fig. 3), diffusely scattered granules in the gray matter neuropil, perivascular aggregations or floccular plaques (Fig. 4). The cytoplasm of ependymal cells or adjacent to affected neuropil also contained $\mathrm{PrP}^{\mathrm{res}}$ positive deposits. The $\mathrm{PrP}^{\mathrm{res}}$ specific band was detected by immunoblotting in the brain and spinal cord sample from the elk (Fig. 5).

Diagnosis of CWD was made based on the results of characteristic brain lesions as well as the detection of PrPres by immunohistochemistry and Western blotting. The pattern and distribution of histologic lesions observed in this case were very similar to those of the previous reports on CWD [7, 11]. Bilaterally symmetrical neuronal vacuolation, spongiform change of the gray matter, and astrocytosis are pathognomonic for CWD. The distribution of $\mathrm{PrP}^{\mathrm{res}}$ in the brain stem was also similar to those of previous reports 
[4]. Immunopositive signal to $\mathrm{PrP}^{\mathrm{res}}$ was most prominent in the dorsal motor nuclei of the vagus nerve. The histological and immunohistochemical diagnosis of CWD was also confirmed at the Animal Diseases Research Institute, Nepean, Canada.

The origin of CWD is not known. However, the disease is thought to be a naturally occurring TSE, comparable to scrapie in sheep, rather than the result of food-borne exposure to the transmissible agent, as in the case of bovine spongiform encephalopathy (BSE) [10]. CWD is transmitted efficiently in nature, with the prevalence reaching $15 \%$ in some wild cervid population in the U.S.A. [2]. It is not certain how CWD is transmitted, but based on epidemiological observations in captive deer species in Colorado and Wyoming, both lateral and vertical transmission may be possible $[1,10]$. CWD has not been naturally transmitted to the other domestic animals.

This CWD positive elk was imported into Korea from a farm in Saskatchewan, Canada to which all other CWD affected premises in Canada have been linked. In addition, several cases of CWD had been diagnosed in the farm of origin of this case (personal communication, Aru Balachandran). In order to eliminate any possibility of horizontal and vertical transmission, all cervids, products and by-products including velvet from the farm in which this elk resided in Korea were destroyed and tested for CWD with negative results.

Korea temporally banned the importation of live elk, deer, and deer products including velvet from the U.S.A. and Canada as of December 28, 2000. Since 1994, a total of 125 elk were imported from the farm occurred CWD in Canada. Soon after the detection of this case, two CWD surveillance programs were initiated by the Korean Ministry of Agriculture and Forestry in 2001. The first program focuses on all imported elk from the source farm and their herd mates. These animals have been under the movement control and quarantine since January 2001, and are subjected to continual monitoring by local veterinarians. All dead elk and deer from these premises, regardless of the presence or absence of clinical signs, are going to be examined for CWD. The other program is geared towards monitoring for
CWD in deer and elk species native to Korea.

Since there are no definitive ante-mortem diagnostic tests for CWD [10]. And the clinical signs of CWD are characteristic but not specific for CWD and are seen in other disease conditions such as malnutrition or wasting systemic diseases. Therefore, greater emphasis is placed on surveillance and testing. Although there is no evidence that CWD could be transmitted to humans, since products of elk and deer such as antler velvet have been widely used in Chinese herbal medicine, it is imperative that well-controlled nationwide surveillance for CWD should be continued.

ACKNOWLEDGMENTS. We thank Chung-San Lee (Chung-buk Livestock and Hygiene Institute) for consultation, and Kim Sun Mi for technical assistance. We also thank all the persons who participated in destruction of all cervids in this farm.

\section{REFERENCES}

1. Miller, M. W., Wild, M. A. and Williams, E. S. 1998. J. Wildl. Dis. 34: 532-538.

2. Miller, M. W., Williams, E. S., McCarty, C. W., Spraker, T. R., Kreeger, T. J., Larsen, C. T. and Thorne, E. T. 2000. J. Wildl. Dis. 36: 676-690.

3. O'Rourke, K. I., Baszler, T. V., Miller, J. M., Spraker, T. R., Sadler-Riggleman, I. and Knowles, D. P. 1998. J. Clin. Microbiol. 36: 1750-1755.

4. Peters, J., Miller, J. M., Jenny, A. L., Peterson, T. L. and Carmichael, K. P. 2000. J. Vet. Diagn. Invest. 12: 579-582.

5. Doi, S., Ito, M., Shinagawa, M., Sato, G., Isomura, H. and Goto, H. 1988. J. Gen.Virol. 69: 955-960.

6. Sigurdson, C. J., Williams, E. S., Miller, M. W., Spraker, T. R., O'Rourke, K. I. and Hoover, E. A. 1999. J. Gen. Virol. 80: 2757-2764.

7. Spraker, T. R., O'Rourke, K. I., Balachandran, A., Zink, R. R., Cummings, B. A., Miller, M. W. and Powers, B. E. 2002. J. Vet. Diagn. Invest. 14: 3-7.

8. Williams, E. S. and Young, S. 1980. J. Wildl. Dis. 16: 89-98.

9. Williams, E. S. and Young, S. 1982. J. Wildl. Dis. 18: 465471.

10. Williams, E. S. and Young, S. 1992. Rev. Sci. Tech. Off. Int. Epizoot. 11: 551-567.

11. Williams, E. S. and Young, S. 1993. Vet. Pathol. 30: 36-45. 